\title{
Manejo híbrido de aneurisma infeccioso de aorta visceral. Caso clínico
}

\author{
RODRIGO SAGÜÉS C., SEBASTIÁN SOTO G.
}

\section{Hybrid management of an infectious pseudoaneurysm. Report of one case}

We report a 61-year-old male with a four months history of progressive back pain, fever, weight loss and hematuria. A CAT scan showed a spondylitis with destruction of L1 and L2 vertebral bodies and a big pseudoaneurysm of the posterior wall of the visceral aorta. A hybrid approach was used to repair the lesion in two stages; a surgical superior mesenteric artery revascularization followed by the placement of an endovascular stent graft in the affected segment of the aorta 48 hours later, excluding the lesion from circulation. In the postoperative period, no evidence of mesenteric vascular insufficiency was detected but the patient developed a systemic inflammatory response that was managed adequately. A CAT scan performed one month later confirmed the exclusion of the pseudoaneurysm. One year after surgery, the patient is able to walk and without evidences of infection or pseudoaneurysm.

(Rev Med Chile 2011; 139: 1071-1074).

Key words: Aneurysm, infected; Prostheses and implants; Stents.
Sección de Cirugía Vascular y Endovascular. Unidad de Enfermedades Cardiovasculares. Hospital Regional de Punta Arenas Dr Lautaro Navarro Avaria.

Recibido el 7 de septiembre de 2010, aceptado el 7 de julio de 2011.

Correspondencia a: Dr. Rodrigo Sagüés C. Paseo Ottawa 0908. Punta Arenas, Chile. Fono (61)233290 E-mail: rsagues@gmail. com

\section{L} os aneurismas infecciosos o "micóticos", corresponden a menos de 1\% de los aneurismas en general. Consisten en la destrucción de la pared arterial por proliferación bacteriana y la formación de un pseudoaneurisma secundario ${ }^{1}$. Su manejo clásico es la antibioterapia, resección del segmento arterial infectado, debridación del tejido subyacente y reemplazo del segmento arterial mediante el uso de injertos autólogos in situ o revascularización extraanatómica ${ }^{1,2}$. Ambas técnicas se asocian a alta morbimortalidad, la cual podría disminuír con el advenimiento de la Cirugía Endovascular; sin embargo, para este tipo de patología la experiencia publicada es anecdótica y se limita sólo a casos clínicos aislados ${ }^{2-5}$. Se presenta caso de un pseudoaneurisma infeccioso de aorta visceral reparado en forma exitosa mediante abordaje híbrido y su seguimiento a un año.

\section{Caso clínico}

Paciente de 61 años, sexo masculino, con antecedentes de etilismo suspendido y sutura de úlcera péptica gástrica perforada a los 50 años de edad. Refería historia de 4 meses de evolución, caracterizada por dolor dorsolumbar progresivo, sensación febril y baja de peso, acompañándose de hematuria en las últimas semanas.

Fue hospitalizado con diagnóstico de pielonefritis aguda recibiendo tratamiento antibiótico empírico a la espera de resultado de urocultivo el cual resultó negativo. Evolucionó febril a pesar de tratamiento antibiótico y con incremento progresivo de dolor dorsal, hasta el punto de ser intratable con analgésicos habituales. Se realizó tomografía axial computada (TAC) de abdomen y pelvis que reveló espondilitis con destrucción de cuerpos vertebrales de L1 y L2 en relación a gran pseudoaneurisma en la cara posterior de la aorta a nivel de tronco celíaco, que se extendía sobre músculo psoas izquierdo hasta nivel de L3 (Figura 1). Se asumió posible etiología infecciosa tanto del pseudoaneurisma como de su espondilitis, se tomaron hemocultivos y se inició tratamiento antibiótico de amplio espectro.

Dada la ubicación de la lesión, clínica de inmi- 


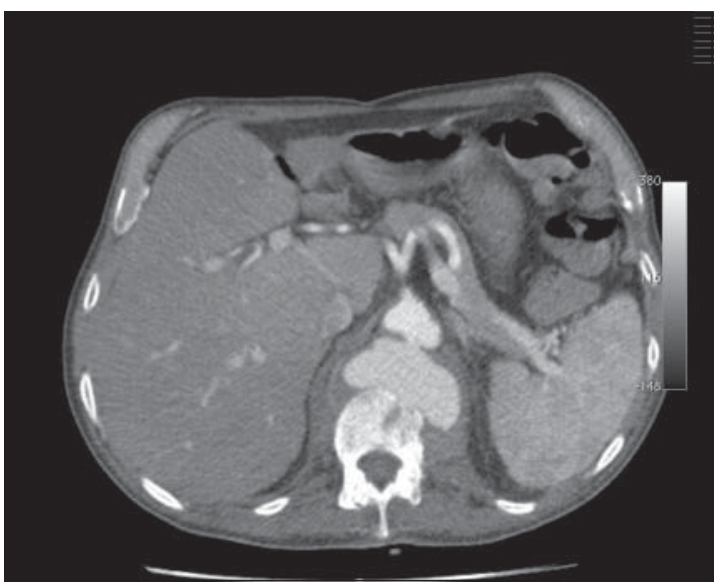

Figura 1. Pseudoaneurisma por cara posterior de aorta visceral en imagen de tomografía axial computada.

nente ruptura y su alto riesgo quirúrgico se evaluó la posibilidad de reparación endovascular, limitada por la necesidad de oclusión de arteria mesentérica superior y tronco celíaco que nacía del área comprometida por el pseudoaneurisma. Se decidió realizar un abordaje híbrido de la lesión, mediante revascularización mesentérica previa a la instalación de endoprótesis recta a nivel de aorta visceral con la finalidad de cubrir el segmento de aorta comprometida y excluir la lesión de la circulación.

En un primer tiempo vía laparotomía media se realizó bypass ilíaco mesentérico superior con vena safena invertida ligando arteria mesentérica proximal, evolucionando sin evidencias de isquemia mesentérica en el postoperatorio inmediato.

En un segundo tiempo, 48 horas posterior a la primera intervención, se realizó aortografía identificando origen del pseudoaneurisma, tronco celíaco, arterias renales y permeabilidad de puente ilíacomesentérico (Figura 2). Luego bajo anestesia local se abordó arteria femoral común izquierda por donde se introdujo extensión de endoprótesis vascular de aorta abdominal Powerlink ${ }^{\circledR}$ (Endologix $^{\circledR}$, California) que se desplegó a nivel de la aorta visceral logrando excluir el pseudoaneurisma, ocluyendo programadamente el tronco celíaco. Se expandió prótesis con balón CODA ( $\operatorname{Cook}^{\circledR}$, Australia), y la angiografía control confirmó la exclusión del pseudoaneurisma y ausencia de endofugas (Figura 3).

En forma inmediata el paciente refirió desaparición del dolor sin mediar analgesia en el

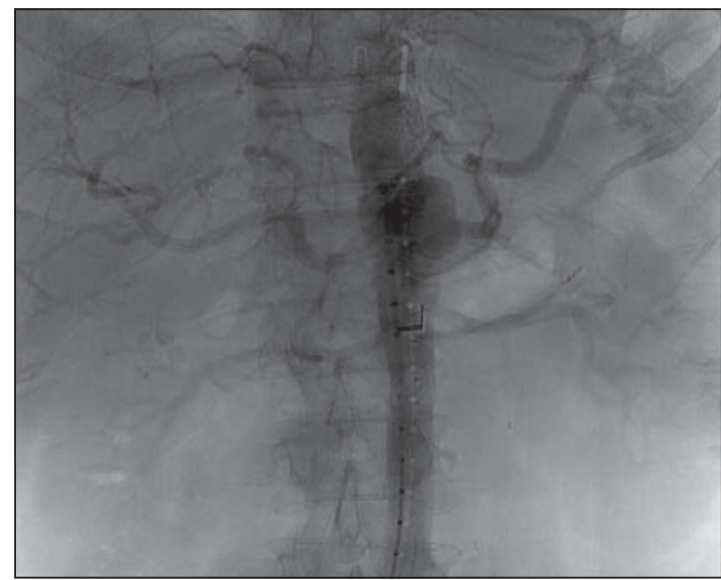

Figura 2. Pseudoaneurisma de aorta visceral en imagen angiográfica.

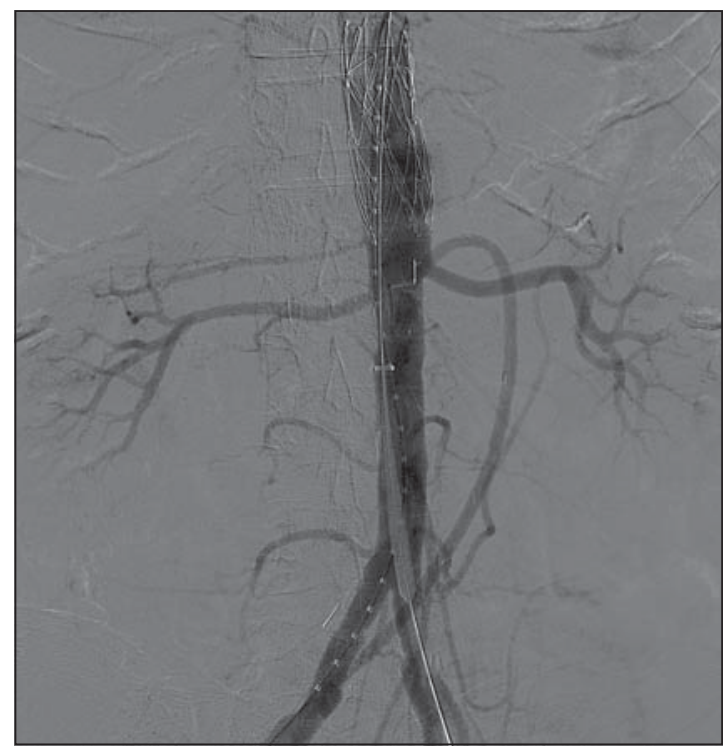

Figura 3. Endoprótesis implantada en aorta visceral excluyendo pseudoaneurisma y bypass ilíaco mesentérico superior permeable.

intraoperatorio. Evolucionó sin evidencias de insuficiencia vascular mesentérica pero con respuesta inflamatoria sistémica asociado a fallas hepática y renal aguda, siendo manejado en UCI con recuperación completa a las $72 \mathrm{~h}$ post procedimiento. Hemocultivos resultaron positivos para Staphylococcus aureus multiresistente; recibió vancomicina por 21 días hasta normalizar parámetros inflamatorios. 


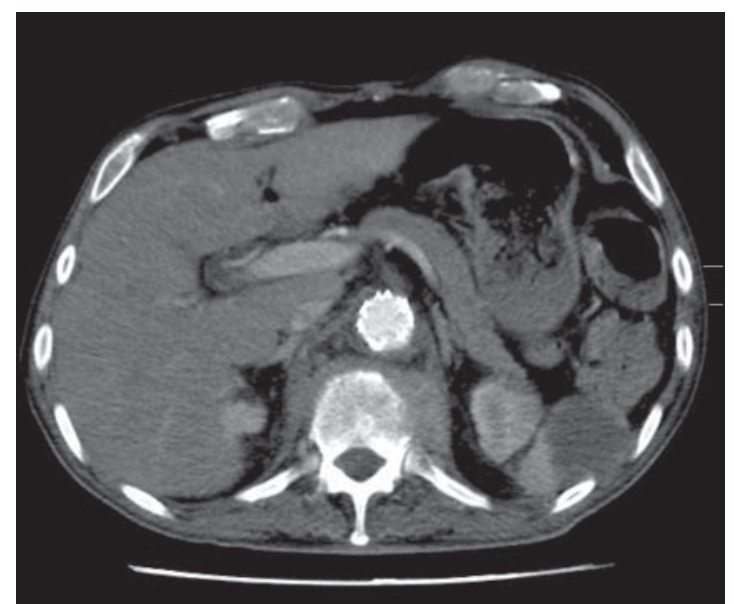

Figura 4. Control tomográfico al mes sin evidencia de endofugas o reproducción del pseudoaneurisma.

Control tomográfico al mes confirmó exclusión del pseudoaneurisma, sin evidencias de isquemia visceral atribuible a la oclusión del tronco celíaco (Figura 4) y revascularización mesentérica permeable; se realizó posteriormente cirugía de columna, en donde no se encontró evidencias de infección. Al año de seguimiento, el paciente se encuentra autovalente y deambulando, sin antibioterapia y sin evidencias clínicas ni de laboratorio de infección, los que se han realizado en forma mensual. Control tomográfico al año de la cirugía no presenta endofugas (Figura 5) y su bypass iliomesentérico está permeable (Figuras 6).

\section{Discusión}

La cirugía endovascular en el territorio aórtico torácico descendente ha logrado posicionarse por sobre la cirugía abierta en la patología aneurismática, dada la menor morbimortalidad asociada especialmente en el período perioperatorio ${ }^{5}$. Distintas series avalan el uso de distintos dispositivos comerciales al igual que técnicas híbridas en caso de requerir ocluir en forma programada ramas viscerales de la aorta ${ }^{5,-7}$.

Los pseudoaneurismas micóticos o infecciosos representan un grupo menor dentro de la patología aneurismática ${ }^{4}$. Su gravedad es mayor dada la alta incidencia de ruptura y la dificultad en su manejo asumiendo que el lecho vascular se encuentra infec$\operatorname{tado}^{1,4,5,8}$. Lo anterior se asocia a tasas de mortalidad de hasta $75 \%$ en distintas series publicadas ${ }^{2}$.

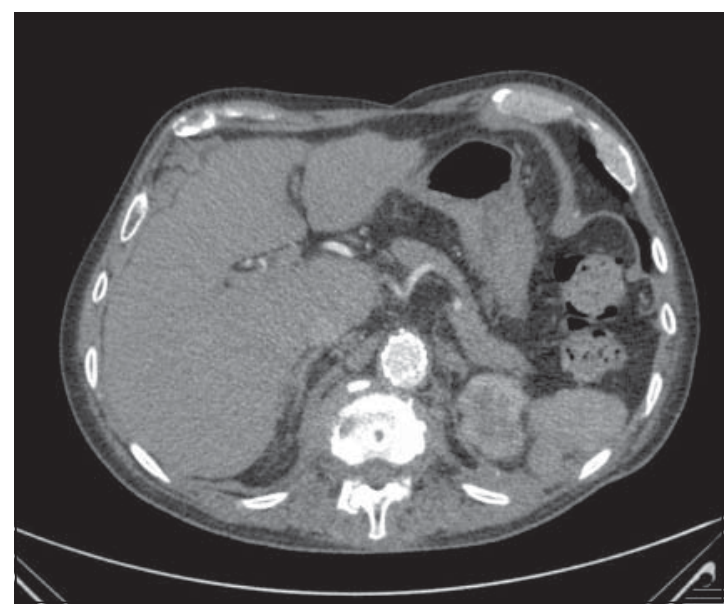

Figura 5. Control tomográfico a un año sin evidencia de endofugas.

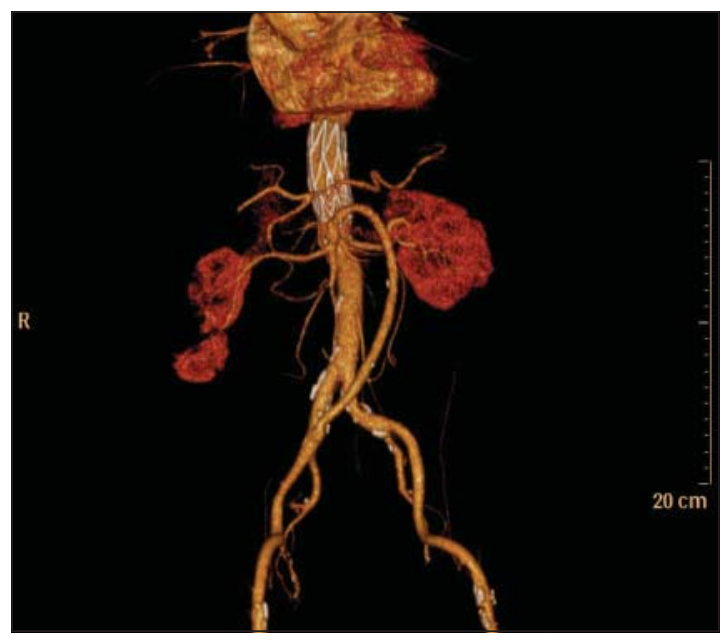

Figura 6. Reconstrucción tomográfica a un año de seguimiento con permeabilidad del bypass y sin evidencia de endofugas.

La utilización de endoprótesis en pseudoaneurismas infecciosos permite obtener los beneficios de la cirugía endovascular, pero implican no resecar ni tratar localmente el territorio afectado, asumiendo la reinfección o infección crónica como una complicación posible a corto y mediano plazo $^{2,9}$.

Dada la baja frecuencia de esta patología, existen escasas publicaciones sobre el tema, sin embargo, logran avalar el uso de la técnica endovascular y concuerdan en la necesidad de vigilancia periódica mediante imágenes y posibles intervenciones 
secundarias ${ }^{3,4,8,9}$, al igual que todo procedimiento endovascular. De igual importancia es la búsqueda mediante imagenología de pseudoaneurismas en otros territorios vasculares, tanto viscerales como de extremidades, que pueden asociarse a la lesión principal y que han sido causa de morbilidad en las distintas series publicadas ${ }^{2,3,4}$.

También es motivo de debate la necesidad o no de antibioterapia post operatoria y la duración de ésta; series de reparación abierta describen crecimiento de cepas bacterianas en cultivos de tejido resecado sólo en $25 \%$ de los casos; de igual modo la frecuencia de hemocultivos positivos no supera el $50 \% \%^{7-9}$. No se reporta mejor sobrevida con diferentes esquemas de duración de antibióticos en los casos de reparación abierta, pero no existe consenso en esta área para la reparación endovascular ${ }^{2,5,8,9}$.

La reinfección o infección crónica se vería favorecida al usar endoprótesis en pacientes con pseudoaneurismas asociados a infección de prótesis previas; en estos casos la sobrevida es menor y debe ser un punto importante al decidir esta técnica ${ }^{2}$. Por otro lado, la presencia de tejido aórtico previamente sano favorecería una mejor sobrevida con menos intervenciones secundarias.

A pesar de que pocas series reportan sobrevida mayor a dos años la mortalidad es principalmente a mediano plazo y habitualmente a causa de las comorbilidades por lo que algunos autores recomiendan el uso de esta técnica en la medida que los dispositivos existentes o la condición del paciente en particular permitan la reparación endovascular ${ }^{2-5,10}$.

\section{Referencias}

1. Müller B, Wegener O, Grabitz K, Pillny M, Thomas L, Sandmann W. Mycotic aneurysms of the thoracic and abdominal aorta and iliac arteries: experience with anatomic and extra-anatomic repair in 33 cases. J Vasc Surg 2001; 33: 106-13.

2. Patel H, Williams D, Upchurch G, Narasimham D, Eliason J, Deeb M. Late outcomes of endovascular aortic repair for the infected thoracic aorta. Ann Thorac Surg 2009; 87: 1366-72.

3. Stanley M, Semmens JB, Lawrence-Brown MM, Denton M, Grosser D. Endoluminal repair of mycotic aneurysms. J Endovasc Ther 2003; 10: 29-32.

4. Jones K, Bell R, Sabharwal T, Aukett M, Reidy J, Taylor P. Treatment of mycotic aortic aneurysms with endoluminal grafts. Eur J Vasc Endovasc Surg 2005; 29: 139-44.

5. Soule M, Javerliat I, Rouanet A, Long A, Lermusiaux P. Visceral debranching and aortic endoprosthesis for a suspected mycotic pseudoaneurysm of the abdominal aorta involving visceral arteries. Ann Vasc Surg 2010; 24: 825e13-825e16.

6. Patel H, Upchurch G, Eliason J, Criado E, Rectenwald J, Williams D, Deeb M. Hybrid debranching with endovascular repair for thoracoabdominal aneurysms: a comparison with open repair. Ann Thorac Surg 2010; 89: 1475-81.

7. Mertens R, Valdés F, Krämer A, Mariné L, Bergoeing M, Sagüés R, et al. Tratamiento "híbrido" del aneurisma tóraco-abdominal: revascularización visceral anatómica e inserción de endoprótesis. Rev Med Chile 2007; 135: 153-9.

8. Kpodonu J, Ramaiah V, Wheatley III G, RodríguezLópez J, Diethrich E. Customized endoluminal graft to treat a suspected saccular thoracoabdominal mycotic aneurysms. Ann Thorac Surg 2008; 85: 1463-5.

9. Koeppel T, Gahlen J, Diehl S, Prosst R, Dueber C. Mycotic aneurysm of the abdominal aorta with retroperitoneal abscess: successful endovascular repair. J Vasc Surg 2004; 40: 164-6.

10. Hsu R, Tsay Y, Wang S, Chu S. Surgical treatment for primary infected aneurysm of the descending thoracic aorta, abdominal aorta, and iliac arteries. J Vasc Surg 2002; 36: 746-50. 